\title{
ACTIVIDAD FÍSICA Y EFECTOS PSICOLÓGICOS DEL CONFINAMIENTO POR COVID-19
}

\author{
Eliseo Andreu Cabrera \\ Profesor Titular de Universidad \\ Departamento de Psicología Evolutiva y Didáctica \\ Facultad de Educación \\ Universidad de Alicante \\ eliseo.andreu@gmail.com
}

Recepción Artículo: 15 abril 2020

Admisión Evaluación: 17 abril 2020

Informe Evaluador 1: 17 abril 2020

Informe Evaluador 2: 119 abril 2020

Aprobación Publicación: 20 abril 2020

\section{RESUMEN}

En diciembre de 2019, la Comisión Municipal de Salud y Sanidad de Wuhan (provincia de Hubei, China), las autoridades chinas identificaron un nuevo tipo de virus denominado COVID 19. La OMS declaró el estado de pandemia mundial y muchos gobiernos decretaron un período de confinamiento prorrogable.

La cuarentena que estamos viviendo, se caracteriza por dos aspectos fundamentales: el aislamiento de nuestros familiares y la restricción de movimiento, tan necesario para el ser humano. Este tiempo de reclusión obligatoria, se ha comprobado que produce daños psicológicos temporales y permanentes. Estrés, irritabilidad y tristeza aparecen ante una situación de soledad, incomunicación e incertidumbre. Este artículo recoge las conclusiones de otros estudios realizados sobre mutaciones del virus SARS y los efectos psicológicos. Algunas investigaciones sugieren que, las duraciones más largas de la cuarentena, se asocian específicamente con síntomas de estrés postraumático, conductas de evitación y enojo. Se analiza la actividad física en el hogar como fenómeno mediático, para luchar contra los desequilibrios emocionales del confinamiento y de mantenimiento de la salud. Ante esta situación de encierro, han proliferado diferentes programas de ejercicios de todo tipo y en diferentes plataformas y medios de comunicación. Algunas de estas plataformas han visto incrementado el número de usuarios y reproducciones, siendo estos valores, indicadores objetivos del interés por la práctica del ejercicio físico en momentos de reclusión obligatoria en el hogar.

Palabras clave: virus; pandemia; cuarentena, efectos psicológicos, salud, deporte, actividad física

\section{ABSTRACT}

Physical activity and psychological effects of confinement by Covid-19. In December 2019, the Wuhan Municipal Health and Sanitation Commission (Hubei Province, China), the Chinese authorities identified a new type of virus called COVID 19. The WHO declared a state of global pandemic and many governments 


\section{ACTIVIDAD FÍSICA Y EFECTOS PSICOLÓGICOS DEL CONFINAMIENTO POR COVID-19}

decreed a period of extendable confinement. The quarantine that we are living, is characterized by two fundamental aspects: the isolation of our relatives and the restriction of movement, so necessary for the human being. This mandatory period of confinement has been proven to produce temporary and permanent psychological damage. Stress, irritability and sadness appear in a situation of loneliness, lack of communication and uncertainty. This article collects the conclusions of other studies carried out on mutations of the SARS virus and the psychological effects. Some research suggests that longer quarantine durations are specifically associated with PTSD, avoidance behaviors, and anger.

Physical activity at home is analyzed as a media phenomenon, to fight against emotional imbalances of confinement and health maintenance. Faced with this situation of confinement, different exercise programs of all kinds have proliferated and on different platforms and the media. Some of these platforms have seen an increase in the number of users and reproductions, these numbers being objective indicators of interest in practicing physical exercise in times of compulsory confinement at home.

Keywords: virus; pandemic; quarantine; psychological effects; health; sport; physical activity

\section{ANTECEDENTES}

El 31 de diciembre de 2019, la Comisión Municipal de Salud y Sanidad de Wuhan (provincia de Hubei, China) informó sobre un agrupamiento de 27 casos de neumonía de etiología desconocida con inicio de síntomas el 8 de diciembre, incluyendo siete casos graves, con una exposición común a un mercado mayorista de marisco, pescado y animales vivos en la ciudad de Wuhan, sin identificar la fuente del brote.

El 7 de enero de 2020, las autoridades chinas identificaron como agente causante del brote un nuevo tipo de virus de la familia Coronaviridae, que ha sido denominado SARS-CoV-2. El Comité de Emergencias del Reglamento Sanitario Internacional (RSI, 2005) declaró el actual brote de nuevo coronavirus como una Emergencia de Salud Pública de Importancia Internacional (ESPII) en su reunión del 30 de enero de 2020. La OMS ha denominado a esta nueva enfermedad COVID-19 (enfermedad infecciosa por coronavirus -19).

Dada la virulencia del nuevo COVID-19 y su reciente aparición, hay pocos estudios científicos que puedan ofrecer datos concluyentes sobre su origen, sintomatología y posible curación. No obstante, algunos de los estudios anteriores sobre los efectos psicológicos del confinamiento, podrían servirnos de referencia a modo de prevención. Junto a la actividad física en el hogar, como fenómeno mundial sobrevenido, se podría mitigar el desgaste emocional de las personas bajo reclusión obligatoria.

\section{OBJETIVOS DE LA INVESTIGACIÓN}

Los objetivos de este trabajo son por un lado, reflejar la situación actual de confinamiento en España, explorando la experiencia de la cuarentena domiciliaria durante el brote del COVID-19. Por otro lado, se pretende hacer un análisis reflexivo sobre los efectos psicológicos que provoca la cuarentena en los seres humanos y de qué manera se buscan rutinas para reducir dichos efectos, mediante la práctica de actividad física en el hogar.

\section{METODOLOGÍA}

La metodología utilizada es de naturaleza cualitativa, por lo que se ha procedido a recoger documentos que nos proveen de datos relativos al problema de la cuarentena por el corona virus y los daños mentales asociados. Se realizó una investigación centrada en el tema de los efectos psicológicos y su mitigación mediante el ejercicio y la actividad física. Paralelamente, dicha investigación se centró en los planes de entrenamiento divulgados por los medios de comunicación, las plataformas digitales e internet. Los datos recogidos de internet fueron objeto de reflexión y discusión, y se realizaron búsquedas en las bases de datos Pubmed,Web of Science, entre otras.

\section{Concepto y características del confinamiento}

Se entiende pandemia como la enfermedad epidémica que se extiende a muchos países o que ataca a casi todos los individuos de una localidad o región. 


\section{CRECIMIENTO PSICOLÓGICO Y AFRONTAMIENTO DE LA MADUREZ}

El término cuarentena proviene de la palabra latina quadraginta (cuatro veces diez), y se usó en Venecia (s. XIV), con las personas sospechosas de tener la peste bubónica y que estaban obligadas a permanecer cuarenta días en aislamiento. En la actualidad, hace referencia al período de reclusión para evitar la propagación de un posible contagio

El período de cuarentena que lamentablemente estamos viviendo, se caracteriza por dos aspectos fundamentales: la separación o aislamiento de nuestros familiares y amigos y la restricción de movimiento, tan necesario para el ser humano. Además supone romper con las rutinas, nuestras aficiones o actividades de ocio y la libertad.

La prolongación de este período provoca el aumento del estrés, debido a muchas causas como el miedo constante a contagiarse, la incertidumbre económica, la pérdida del trabajo, la reducción de ingresos mensuales, la frustración provocada por el sedentarismo forzado, convivir todo el día con la pareja, hijos o compañeros de piso.

El aislamiento supone separarse de las personas para evitar ser contagiado o contagiar a terceras personas. Además, afecta al ser humano de manera diferente según su edad. Por ello, el impacto psicológico no será el mismo en niños, que en adultos solteros/separados, que en adultos que conviven en familia, o que en personas de la tercera edad que ya sufrían la soledad y el aislamiento social, antes de la pandemia.

Este tiempo de reclusión obligatoria, se ha comprobado que produce daños psicológicos temporales y permanentes. Estrés, confusión, irritabilidad y tristeza aparecen ante una situación de soledad, incomunicación, desinformación e incertidumbre. La excesiva exposición a noticias negativas, así como la saturación de los centros médicos son también causas del estrés y la ansiedad.

\section{La cuarentena en España}

El 14 de marzo de 2020, el Gobierno español decretó el estado de alarma, y al objeto de impedir la propagación del coronavirus, ordenó el confinamiento de todos los españoles.

Se ha comprobado que los períodos de cuarentena suelen causar daño mental en los individuos, ya que entre otras cosas, somos seres sociales que necesitamos la comunicación interpersonal. No obstante, de esa convivencia por exceso, inusual y continua, también surgirán situaciones estresantes por falta de espacio vital.

Las recomendaciones que realizan los gobiernos para que no salgamos de casa, en ocasiones se viven como auténticas amenazas y hacen sentir a la persona, como un proscrito aunque vaya a la panadería de la esquina. Surgen los "policías de balcón", la intransigencia hacia los otros, fruto de la frustración personal.

Paralelamente al problema de salud, la incertidumbre económica ante el cierre temporal de las empresas, el descenso en la actividad laboral, las caídas de la Bolsa, suponen otra preocupación añadida que causa desórdenes psicológicos. El posible desabastecimiento en los comercios y el pánico generalizado, puede causar problemas de rebelión en las calles.

En España, el numero de muertes ha ido incrementándose paulatinamente, hasta el punto de alcanzar a fecha 21 de abril de 2020, un total de 21.717 fallecidos y 208.389 contagiados (Ministerio de Sanidad. Gobierno de España).

Por zonas, parece que la mayor concentración de contagios se está produciendo en el centro de la península, siendo Madrid, la comunidad autónoma con más casos (aproximadamente 60.000). 


\section{ACTIVIDAD FíSICA Y EFECTOS PSICOLÓGICOS DEL CONFINAMIENTO POR COVID-19}

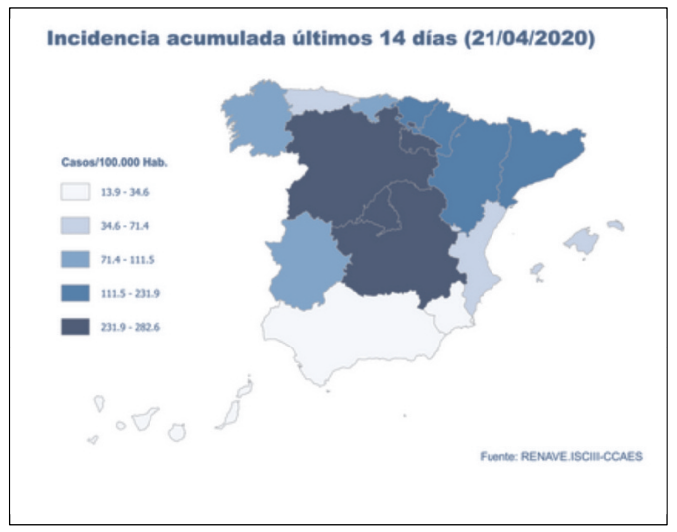

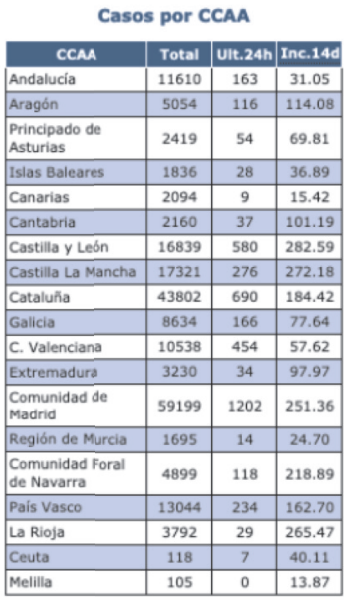

Como medidas de prevención, el Ministerio de Sanidad, realizó una serie de recomendaciones que se basaban en el lema "Quédate en casa". Entre otras, se aconsejaba mantener distancias de dos metros entre los convivientes, evitar visitas en el domicilio, usar productos de higiene de manos y usar mascarillas y guantes para prevenir contagios.

Entre las normas de limpieza, se recomendó fregar con agua caliente $\left(70^{\circ}\right)$, meter la ropa usada en una bolsa y limpiar las superficies con lejía diluida.

\section{Relevancia del tema: Ios efectos psicológicos de la cuarentena}

Muchos de los estudios revisados destacan efectos psicológicos negativos. Entre otros, aparecen temores de infección, frustración, aburrimiento, suministros inadecuados, información inadecuada, pérdidas financieras y estigma. Algunos investigadores han sugerido efectos duraderos, incluso hasta tres años después de la cuarentena por el virus (SARS).

Cava, M.A. et al. (2005), realizaron un estudio en 2003, titulado "The Experience of Quarantine for Individuals Affected by SARS in Toronto". El objetivo fue explorar la experiencia de la cuarentena domiciliaria durante el brote del síndrome respiratorio agudo severo (SRAS) en Toronto en 2003. La experiencia siguió una trayectoria de etapas que comienzan antes de la cuarentena y terminan después de la cuarentena. Como conclusiones, afirmaron que la salud pública tiene una doble función: de monitorear el cumplimiento y brindar apoyo a las personas en cuarentena. Este estudio tiene implicaciones para la política y la práctica de salud pública en la planificación de futuras emergencias de salud pública en términos de la información y los recursos necesarios para organizar una respuesta efectiva.

Algunas investigaciones sugieren que, las duraciones más largas de la cuarentena, se asocian específicamente con una peor salud mental, síntomas de estrés postraumático, conductas de evitación y enojo.

Otro estudio muy relevante de estos mismos autores, Cava, M.A. et al. (2005), titulado "Risk perception and compliance with quarantine during the SARS outbreak". En él analizaron la experiencia de estar en cuarentena para el síndrome respiratorio agudo severo (SRAS) con un enfoque en la relación entre el riesgo percibido de contraer el SRAS y el cumplimiento informado de la orden y los protocolos de cuarentena.

Como resultados, comprobaron que los participantes en el estudio evolucionaron entre el miedo y la negación, sobre su riesgo de contraer o propagar el SRAS. Sobre el cumplimiento de las normas de la cuarentena, se concluyó que fue alto, sin embargo, dentro de los hogares los protocolos de cuarentena se siguieron de manera desigual. Esta investigación indicó la necesidad de una mayor credibilidad en las comunicaciones de salud 
pública para aumentar el cumplimiento de los protocolos de cuarentena y contener brotes de enfermedades infecciosas nuevas y mortales.

The Lancet, ha publicado recientemente), diferentes estudios sobre la perspectiva planetaria del virus, desarrollo de test basados en anticuerpos, inmunidad y anticuerpos, formas de transmisión, entre otros. Cabe destacar el artículo de Betsch C. et al (2020), que recoge un análisis de los comportamientos psicológicos asociados al virus y su origen, tales como comportamiento de rebaño (acaparamiento de alimentos o papel higiénico) o la estigmatización de las personas contagiadas.

Mientras las revistas más prestigiosas se centran actualmente en la solución de esta pandemia, (medicación y vacunas), debemos recurrir a los estudios realizados con anterioridad sobre los efectos psicológicos del virus SARS, similar al COVID-19. Uno de los estudios más relevantes es "SARS Control and Psychological Effects of Quarantine", de Hawryluck, L. et al. (2004).

Por otra parte, los datos que nos Ilegan desde Wuhan (China), apuntan a que el 40\% de las personas aisladas han sentido algún síntoma de ansiedad.

Otro interesante y reciente artículo, es el publicado por Brooks, S.K. et al. (2020), en la prestigiosa revista The Lancet, bajo el título "The psychological impact of quarantine and how to reduce it: rapid review of the evidence". Estos autores concluyen que el impacto psicológico de la cuarentena es amplio, sustancial y puede ser duradero. No obstante, defienden la cuarentena como método de contención del virus. Por otra parte, afirman que privar a las personas de su libertad para el bien público en general es a menudo polémico y debe manejarse con cuidado.

Todas las medidas que se tomen desde los gobiernos, deben garantizar que esta experiencia sea lo más tolerable posible para las personas. Esto se puede lograr: diciéndole a las personas lo que está sucediendo y por qué, explicando cuánto tiempo continuará, proporcionando actividades significativas para que realicen mientras están en cuarentena, proporcionando una comunicación clara, asegurando suministros básicos (como alimentos, agua y suministros médicos) están disponibles y refuerzan la sensación de altruismo que las personas deberían, con razón, sentir. Concluyen finalmente que si la experiencia de la cuarentena es negativa, puede haber consecuencias a largo plazo, tanto de salud como económicas.

Entre los profesionales de la Psicología se aconseja llevar a cabo rutinas diarias para evitar el desgaste psicológico que puede causar el aislamiento social. Según el doctor, el orden mental nos ayudará a estar más tranquilos, equilibrados y seguros. El aislamiento supone la falta de libertad de movimiento y la incomunicación, por lo que cierta disciplina física podría ayudar a reducir la angustia vital y otros efectos del confinamiento. No hay que olvidar, utilizar la tecnología para buscar situaciones de comunicación interpersonal y luchar contra la soledad.

\section{La cuarentena y los efectos psicológicos}

Según los estudios analizados, sus autores parecen coincidir en que los efectos psicológicos del confinamiento son:

En la población en general:

La ansiedad y el miedo provocados por la sensación de vivir una catástrofe mundial.

Incertidumbre ante el futuro, pues no se sabe lo que va a ocurrir y no tenemos el control de la situación.

La frustración personal, que nos Ileva a la apatía 0 a la búsqueda constante de información actualizada, lo que provoca ansiedad de estado. La exposición al exceso de información en medios de comunicación, internet, y aplicaciones populares como WhatsApp, ha generado otro fenómeno: la propagación de bulos e informaciones sin contrastar, muchas veces por inercia. El exceso de información sobre los síntomas del COVID-19, también provoca comportamientos hipocondríacos.

Durante la convivencia se sufren altibajos emocionales que afectan a las relaciones personales.

Otro consecuencia, es la soledad, sobre todos de las personas mayores. El ser humano necesita comunicarse con otras personas y contactar para transmitir sentimientos e ideas. Cuando se sufre la privación de este contacto, surge la tristeza y la depresión. 


\section{ACTIVIDAD FÍSICA Y EFECTOS PSICOLÓGICOS DEL CONFINAMIENTO POR COVID-19}

En la población infantil:

En el caso de los niños, ciertos estudios afirman que las secuelas psicológicas o traumas tras la pandemia, podrían caracterizarse por síntomas de estrés postraumático, regresiones a etapas anteriores, agresividad y rebeldía, o incluso desórdenes en el sueño. Hay que tener en cuenta, que los niños absorben el clima emocional que trasmiten sus padres en el hogar.

Otro efecto fisiológico es la obesidad, ya que se descuida la alimentación y se busca un remedio contra la ansiedad.

El confinamiento también agravará las patologías preexistentes al período de reclusión en los hogares.

Los niños tendrán problemas en la regulación emocional y conductual, pues se encuentra en pleno desarrollo y sufren una privación de movimiento, juego en la calle e interacción social. Además, necesitan rutinas y hábitos para sentirse seguros, lograr la estabilidad y seguir su ritmo biológico.

La restricción de movimiento provocará enfados, Ilantos, miedos, trastornos alimentarios y cierta hiperactividad

\section{Estrategias para contrarrestar los efectos del confinamiento}

\section{A nivel estatal}

Como apuntan los estudios más recientes, para enfrentar un período de cuarentena, algo muy importante es el suministro de información a los ciudadanos, de material sanitario y de alimentos. El período de confinamiento debe ser corto y no alargarse más allá de lo necesario, aprovechando el tiempo para realizar tests a la población con el objetivo de controlar el número de casos y evitar el colapso del sistema de salud.

La restricción de libertades puede acabar en la rebelión de los ciudadanos, por lo que la recomendación de autoaislamiento puede ser más efectiva (tal y como se ha realizado en países como Alemania o Dinamarca).

Atención especial a los médicos y enfermeros. Ciertos estudios demuestran que existe alta prevalencia de angustia psicológica en los trabajadores de la salud en cuarentena, por lo que habría que darles prioridad en cuanto a la dotación de material sanitario de seguridad.

Facilitar una red de teleoperadores para ofrecer información a los confinados y facilitar las gestiones de tipo administrativo.

\section{A nivel personal}

Mantener la comunicación visual y verbal con nuestros amigos y familiares, manteniendo las precauciones básicas y evitando la estigmatización.

Evitar la saturación de noticias negativas y ceñirse a fuentes oficiales, huyendo de bulos y falsedades.

Establecer rutinas y actividades (aseo personal, ejercicio, alimentación, sueño,...) en el día a día, pues estar ocioso está prohibido.

Tener espacios y tiempos para uno mismo, dentro de casa.

Mantener el sistema inmunológico fuerte mediante una alimentación saludable y suplementos vitamínicos como la vitamina C. La depresión puede debilitar nuestras defensas.

Utilizar internet y las tecnologías de la comunicación para entretenernos, socializar y mantener encuentros virtuales con familiares y amigos.

Luchar contra el sedentarismo realizando actividad física en el hogar.

\section{Específicas para la población infantil}

Promover actividades lúdicas durante el día

Utilizar la música como recurso

Controlar el tiempo de uso de los dispositivos digitales, evitando su uso excesivo

Aprovechar la situación de confinamiento para aumentar el tiempo y calidad de la comunicación con los más pequeños. 
Establecer rutinas, horarios y hábitos básicos

\section{La actividad física en época de confinamiento}

La Organización Mundial de la Salud recomienda 150 minutos de actividad física moderada por semana, 10 que supone unos 30 minutos diarios. La actividad física moderada se convierte en la mejor inversión en salud para un país.

Numerosas universidades han querido analizar los efectos del confinamiento entre el alumnado universitario. En España, destaca el proyecto liderado por la universidad de Granada, y en el que participan 15 universidades españolas, denominado "Actividad física en la población universitaria durante el confinamiento por Covid-19: determinantes y consecuencias sobre el estado de salud y la calidad de vida" (2020), que pretende conocer cómo adapta el alumnado sus hábitos de actividad física y estilo de vida a la situación de confinamiento. Según los expertos, los resultados permitirán diseñar estrategias para hacer frente a las consecuencias negativas, tanto para el periodo posterior al confinamiento, como para situaciones similares que puedan ocurrir en el futuro.

Para un ciudadano poco entrenado, la actividad física y el deporte es algo más que un hábito de catarsis, ya que podría convertirse en la salvación ante la enfermedad. La actividad física se presenta como un verdadero aliado en la lucha contra el virus y contra sus efectos psicológicos.

Diversos estudios demuestran la efectividad de la actividad física en el fortalecimiento del sistema inmunitario. Destaca el reciente estudio de Sallis, J. And Pratt, M. (2020), titulado "A Call to Action: Physical Activity and COVID-19" y publicado por el American College of Sports Medicine. En él, se afirma que la actividad física moderada podría ayudar a reducir la inflamación en los pulmones producida por el COVID-19.

También defienden que la actividad física podría prevenir enfermedades cardíacas, diabetes y neoplasias, las cuales aumentan el riesgo de muerte entre las personas infectadas con el coronavirus.

Paralelamente, estos autores propugnan que cada sesión de actividad física reduce los síntomas de depresión y ansiedad. Citando los estudios de Powell et al.(2019) sostienen que estar activo es tan efectivo como los medicamentos y la psicoterapia.

Entre otras cosas, la cuarentena nos ha enseñado que podemos practicar actividad física sin necesidad de ir a un gimnasio. Esto ha generado un verdadero fenómeno en las redes sociales y ha acabado con las existencias de material deportivo en los grandes almacenes que venden por internet.

Como respuesta social a la restricción de movimiento, se ha producido un fenómeno deportivo nunca antes visto: La actividad física en el hogar.

Podemos constatar una gran proliferación de tablas de ejercicios físicos en las redes sociales, medios de comunicación, canales de Youtube e instituciones o entidades de todo tipo.

En cuanto a los videojuegos de ejercicio físico, más vendidos en época de pandemia, destaca Ring Fit Adventures (Nintendo Switch), que se ha agotado en varios países y ha disparado su precio hasta los 159 euros. Otro algo más antiguo, de la plataforma Wii es el Wii Fit. En PS4, destaca Aces of the Multiverse orientado a la actividad física en niños. En la plataforma Xbox, el más exitoso es Zumba Fitness World Party.

Los gimnasios, que se ha visto obligados a cerrar, lanzan aplicaciones de teléfono en las que se ofrecen tablas personalizadas, con el fin de fidelizar a futuros clientes. En Instagram se ha creado una cuenta a raíz de una iniciativa de distintos entrenadores personales: \#YoMeMuevoEnCasa.

En Youtube, como apuntábamos anteriormente, existen numerosos canales, entre los que sobresale Gymvirtual, con más de 7,5 millones de suscriptores y los vídeos acumulan más de 10 millones de visualizaciones. El canal Xuan Lan Yoga posee 507.000 suscriptores.

Entre otros, resaltamos las siguientes ofertas de Youtube para la actividad física en el hogar, a fecha 23/4/2020: 
En español:

\begin{tabular}{|c|c|c|}
\hline TÍTULO & ENLACE & $\begin{array}{l}\mathrm{N}^{\mathrm{o}} \quad \mathrm{DE} \\
\text { VISUALIZACION } \\
\mathrm{ES} \\
\end{array}$ \\
\hline $\begin{array}{l}\text { COVID19 Tutorials - Educación } \\
\text { Física (Infantil y Primaria) }\end{array}$ & $\begin{array}{l}\text { https://www.youtube.com/watch?v } \\
=\mathrm{Gn} 7 \mathrm{k} \text { xNXYBc }\end{array}$ & 257.000 \\
\hline $\begin{array}{ll}\text { \#QuedateEncasa RUTINA } & \text { DE } \\
\text { CARDIO INTENSO } & \text { EN } \\
\text { CUARENTENA } & \\
\text { \#EntrenaConmigo } & \\
\end{array}$ & $\begin{array}{l}\text { https://www.youtube.com/watch?v } \\
=\text { TbXsX RkI2o }\end{array}$ & 162.000 \\
\hline $\begin{array}{l}\text { Juegos divertidos para niños en } \\
\text { CASA. COVID. } 19\end{array}$ & $\begin{array}{l}\text { https://www.youtube.com/watch?v } \\
=\text { QHMk0MUJcEA }\end{array}$ & 85.000 \\
\hline $\begin{array}{l}\text { CUIDADO! Entrenar podría } \\
\text { bajar tus defensas | Qué hacer } \\
\text { ante el Coronavirus }\end{array}$ & $\begin{array}{l}\text { https://www.youtube.com/watch?v } \\
=\text { q9mi -DZyL8 }\end{array}$ & 63.000 \\
\hline $\begin{array}{lrr}\text { Las mejores } & \text { ACTIVIDADES y } \\
\text { JUEGOS para } & \text { una } \\
\text { EDUCACIÓN FÍSICA } & \text { EN } \\
\text { CASA, ideal en tiempos de } \\
\text { CORONAVIRUS! } & \\
\end{array}$ & $\begin{array}{l}\text { https://www.youtube.com/watch?v } \\
=\text { WJAeCjFpQHM }\end{array}$ & 43.000 \\
\hline $\begin{array}{l}\text { Actividad Física durante la } \\
\text { cuarentena \#Coronavirus }\end{array}$ & $\begin{array}{l}\text { https://www.youtube.com/watch?v } \\
=\text { oSdIpxcNXIM }\end{array}$ & 33.000 \\
\hline $\begin{array}{l}\text { EDUCACIÓN FÍSICA } \\
\text { INFANTIL - CLASE EN CASA } \\
\text {-\#MeQuedoEnCasa }\end{array}$ & $\begin{array}{l}\text { https://www.youtube.com/watch?v } \\
\text { =nIZfpfi-ENE }\end{array}$ & 19.000 \\
\hline $\begin{array}{l}\text { Juegos, actividades y dinámicas } \\
\text { para una EDUCACIÓN FISICA } \\
\text { en CASA y SIN MATERIAL } \\
\text { lucha vs COVID-19 }\end{array}$ & $\begin{array}{l}\text { https://www.youtube.com/watch?v } \\
=\mathrm{e} 66 \mathrm{fMh} 4 \mathrm{iOuc}\end{array}$ & 10.000 \\
\hline $\begin{array}{l}\text { JUEGOS y ACTIVIDADES de } \\
\text { EDUCACIÓN FISICA EN } \\
\text { CASA con } \\
\text { CASERO ideal parERIAL } \\
\text { alumnos|Covid-19 }\end{array}$ & $\begin{array}{l}\text { https://www.youtube.com/watch?v } \\
=34 \mathrm{wIS6NMRXA}\end{array}$ & 10.000 \\
\hline $\begin{array}{l}\text { Hábitos saludables durante el } \\
\text { estado de alarma (COVID-19) }\end{array}$ & $\begin{array}{l}\text { https://www.youtube.com/watch?v } \\
\text { =AaR3Wjcvpv0 }\end{array}$ & 9.000 \\
\hline $\begin{array}{llr}\text { EJERCICIOS para hacer } & \text { en } \\
\text { CASA durante } & \text { el } \\
\text { CONFINAMIENTO } & \text { del } \\
\text { CORONAVIRUS } & \\
\end{array}$ & $\begin{array}{l}\text { https://www.youtube.com/watch?v } \\
=\mathrm{D} 2 \mathrm{XzTaJBrcw}\end{array}$ & 6.000 \\
\hline $\begin{array}{l}\text { EJERCICIO SEGURO } \\
\text { CONFINAMIENTO: } \\
\text { CLAVES para tu SUELO } \\
\text { PÉLVICO }\end{array}$ & $\begin{array}{l}\text { https://www.youtube.com/watch?v } \\
=3 \text { IwlQPGQMd8 }\end{array}$ & 6.000 \\
\hline
\end{tabular}


En inglés:

\begin{tabular}{|c|c|c|}
\hline TÍTULO & ENLACE & $\begin{array}{l}\mathrm{N}^{\mathrm{o}} \mathrm{DE} \\
\text { VISUALIZACION } \\
\text { ES }\end{array}$ \\
\hline $\begin{array}{l}\text { Quarantined Home?? } 20 \text { minute } \\
\text { exercise routine for seniors and } \\
\text { beginners }\end{array}$ & $\begin{array}{l}\text { https://www.youtube.com/watch?v } \\
=\mathrm{AE} \mathrm{FeKpc} \mathrm{lk}\end{array}$ & 702.000 \\
\hline $\begin{array}{l}\text { Training In Quarantine For } 3 \\
\text { Weeks }\end{array}$ & $\begin{array}{l}\text { https://www.youtube.com/watch?v } \\
=\text { eUIGK7jLjEM }\end{array}$ & 500.000 \\
\hline $\begin{array}{l}\text { Jacqueline Fernandez Motivate } \\
\text { Exercise in Home Quarantine } \\
\text { during Lockdown | Fitness } \\
\text { Challenge }\end{array}$ & $\begin{array}{l}\text { https://www.youtube.com/watch?v } \\
=\text { AUA-XrMDYnE }\end{array}$ & 166.000 \\
\hline $\begin{array}{l}\text { Things to do during Quarantine : } \\
\text { Home Workout, Meditation, } \\
\text { Yoga \& more }\end{array}$ & $\begin{array}{l}\text { https://www.youtube.com/watch?v } \\
=\text { lpUzwtNx6cQ }\end{array}$ & 121.000 \\
\hline $\begin{array}{l}\text { Exercise you can do at home } \\
\text { during the coronavirus outbreak }\end{array}$ & $\begin{array}{l}\text { https://www.youtube.com/watch?v } \\
=\text { GQClmVgpUu4 }\end{array}$ & 10.000 \\
\hline $\begin{array}{l}\text { Physical activity session for } \\
\text { Beginners during self-quarantine }\end{array}$ & $\begin{array}{l}\text { https://www.youtube.com/watch?v } \\
=\text { zcZiuUuzYcA }\end{array}$ & 4.000 \\
\hline
\end{tabular}

En cuanto al material disponible en forma de guía o recomendaciones, destacan las siguientes:

Para la población infantil:

Colegio de licenciados en Educación Física

https://www.consejo-colef.es/recursosef-covid19-primaria

Para la población en general

http://www.euro.who.int/en/health-topics/health-emergencies/coronavirus-covid-19/novel-coronavi-

rus-2019-ncov-technical-guidance/stay-physically-active-during-self-quarantine/_recache\#article

https://www.easp.es/coronavirus-easp/

https://psiquiatria.com/corona-virus-covid-19/recomendaciones-actividad-fisica-covid-19/

https://www.cfisiomad.org/Pages/detalle_noticia_pub.aspx?idnoticia=1960

Para la tercera edad:

https://www.pdmarrecife.es/guia-de-actuacion-en-confinamiento-y-actividad-fisica-para-personasmayores-covid-19/

https://fundaciondescubre.es/noticias/disenan-una-guia-para-hacer-actividad-fisica-en-casa-dirigidaa-personas-mayores/

https://www.20minutos.es/noticia/4208294/0/coronavirus-la-diputacion-facilita-una-guia-para-quepersonas-mayores-hagan-ejercicio-en-casa/

\section{DISCUSIÓN}

El confinamiento impuesto por el gobierno de España tiene como objetivo evitar la propagación de la pandemia. Dicha medida tan extrema solamente puede llevarse a cabo con la colaboración de la población. Cierto es que el período de reclusión no debe ser muy amplio para que los efectos psicológicos no se agraven y la economía del país no se resienta. La mayoría de los estudios apuntan a efectos negativos para la salud mental de la población, tanto durante como después de la pandemia. Al miedo por el contagio, se añaden el estrés, la frustra- 


\section{ACTIVIDAD FÍSICA Y EFECTOS PSICOLÓGICOS DEL CONFINAMIENTO POR COVID-19}

ción, la incertidumbre financiera, la estigmatización o el aislamiento social. Como método para mitigar ciertos efectos psicológicos, aparece la actividad física en el hogar como fenómeno espontáneo. Proliferan entrenadores personales a distancia, guías y tablas virtuales para vencer el aburrimiento, el estrés y favorecer el mantenimiento de la salud.

\section{CONCLUSIONES}

La cuarentena es un medio para luchar contra la pandemia, por lo que los gobiernos priorizan controlar el virus, ante una situación de deterioro mental de los ciudadanos. Los efectos psicológicos pueden tratarse, pero una expansión del virus sería letal para la comunidad.

No obstante, la cuarentena debe tener un calendario de regulación y no extenderse más allá de lo necesario. Actualmente, han surgido gran número de querellas contra el gobierno por privación de libertades, pues la población no entiende el estado de alarma como un estado de sitio. Quizás, el gobierno debería proporcionar información regularmente, para que no influyan los bulos y provoquen confusión de los ciudadanos. No existen muchos estudios para evaluar las secuelas psicológicas de la cuarentena por COVID-19.

Algunas investigaciones presentan la actividad física de mantenimiento y las actividades lúdicas, como una excelentes herramientas para luchar contra los efectos psicológicos del confinamiento. Esta idea parece haber calado en la población mundial y prueba de ello es la cantidad de programas de actividad física para el hogar y el gran número de practicantes en todo el mundo.

\section{REFERENCIAS BIBLIOGRÁFICAS}

Betsch, C., Wieler, L.H., Habersaat, K. (2020). Monitoring behavioural insights related to COVID-19. Lancet 395 (10232), P1255-1256.

Brooks, S. K., Webster, R. K., Smith, L. E., Woodland, L., Wessely, S., Greenberg, N. y Rubin, G. J. (2020). The psychological impact of quarantine and how to reduce it: Rapid review of the evidence. Lancet, 395 (10227), 912-920.

Cava, M.A., Fay, K.E., Beanlands, H.J., McCay, E.A., Wignall, R. (2005). The experience of quarantine for individuals affected by SARS in Toronto. Public Health Nurs. 2005; 22: 398-406

Cava, M.A., Fay, K.E., Beanlands, H.J., McCay, E.A., Wignall, R. (2005). Risk perception and compliance with quarantine during the SARS outbreak. Public Health Nurs. 2005; 37(4): 343-347

Duan, L. y Zhu, G. (2020). Psychological interventions for people affected by the COVID-19 epidemic. Lancet Psychiatry, 7(4), 300-302. https://doi.org/10.1016/S2215-0366(20)30073-0

Hawryluck, L. et al. (2004). SARS Control and Psychological Effects of Quarantine. Emerging Infectious Diseases. 10 (7): 1206-1212

Inchausti, F., García-Poveda N.V., Prado-Abril J. y Sánchez-Reales S. (2020). La Psicología Clínica ante la Pandemia COVID-19 en España. Clínica y Salud. Avance online. https://doi.org/10.5093/clysa2020a11

Organización Mundial de la Salud (OMS) (2020). Coronavirus disease (COVID- 19). https://www.who.int/emergencies/diseases/novel-coronavirus-2019/

Sallis, J. y Pratt, M. (2020). A Call to Action: Physical Activity and COVID-19. American College of Sports Medicine. Disponible en: https://www.exerciseismedicine.org/support_page.php/stories/?b=896

https://www.psicologiamadrid.es/blog/articulos/psicologia-y-coronavirus/coronavirus-efectos-psicologicosgenerados-por-el-confinamiento

https://elmedicointeractivo.com/el-confinamiento-produce-efectos-psicologicos-negativos-como-estres-postraumatico-confusion-y-enfado/

https://www.intramed.net/contenidover.asp?contenidoid=95688

https://www.larazon.es/salud/20200418/60lg4xllvzbrpayqazwcadd4ra.html

https://retina.elpais.com/retina/2020/04/03/talento/1585929680_702574.html 
https://www.sciencedirect.com/science/article/pii/S0140673620304608\#!

https://faros.hsjdbcn.org/es/articulo/efectos-ninos-confinamiento-casa-coronavirus-medidas-prevencion-actuacion

https://www.cambio16.com/efectos-del-confinamiento/

https://www.sunrisemedical.es/blog/coronavirus-efectos-negativos-del-confinamiento-en-la-tercera-edad

https://www.efesalud.com/efectos-psicologicos-confinamiento-consejos/

https://theconversation.com/los-efectos-del-confinamiento-en-los-deportistas-de-elite-134917

https://uniandes.edu.co/es/noticias/psicologia/la-actividad-fisica-es-fundamental-en-tiempos-de-coronavirus 
